\title{
On The Similarity State Transformation for Linear Parameter-Varying Systems
}

\author{
Balázs Kulcsár* Roland Tóth ** \\ * Department of Signals and Systems, Chalmers University of \\ Technology, SE-412 96, Gothenburg, Sweden, kulcsar@chalmers.se. \\ ** Delft Center for Systems and Control, Delft University of \\ Technology, Mekelweg 2, 2628 CD Delft, The Netherlands, \\ r.toth@tudelft.nl.
}

\begin{abstract}
Similarity state transformations between equivalent State-Space (SS) representations of discrete-time Linear Parameter-Varying (LPV) systems are investigated. Based on previous results, it is known that to characterize all equivalent LPV-SS representations, the statetransformation matrix must depend dynamically on the scheduling variable. However, preserving static dependence of a LPV-SS representation, i.e. characterizing all equivalent SS representation with static dependence, has primary importance both in control and identification. Therefore, first, the state transformation problem is investigated from an algebraic (behavior) point of view, then conditions are developed to guarantee preservation of the static dependence in similarity state transformations. Additional geometric interpretation of the obtained results, together with computational approach to synthesize state-transformations are also developed. An illustrative example is provided to demonstrate the validity of the obtained results.
\end{abstract}

Keywords: Linear parameter-varying systems, similarity state transformation.

\section{INTRODUCTION}

In linear parameter-varying (LPV) control, e.g. Scherer (1996); Apkarian and Gahinet (1995) and in state-space (SS) identification e.g. Lee and Poolla (1996); Felici et al. (2006), discrete-time LPV systems are often described in terms of LPV-SS representations, commonly defined in the following form:

$$
\begin{aligned}
x(k+1) & =A(p(k)) x(k)+B(p(k)) u(k), \\
y(k) & =C(p(k)) x(k)+D(p(k)) u(k),
\end{aligned}
$$

where $u: \mathbb{Z} \rightarrow \mathbb{R}^{n_{\mathrm{u}}}, y: \mathbb{Z} \rightarrow \mathbb{R}^{n_{\mathrm{y}}}$ and $x: \mathbb{Z} \rightarrow \mathbb{R}^{n_{\mathrm{x}}}$ are the input, output and state signals of the system respectively. The system matrices $A, B, C, D$ are rational functions of the scheduling signal $p: \mathbb{Z} \rightarrow \mathbb{P}$ and are bounded on $\mathbb{P}$, where the conected set $\mathbb{P} \subseteq \mathbb{R}^{n_{\mathrm{p}}}$ is the so called scheduling 'space'. $p$ is considered as an external, i.e. independent signal of the system. However in applications, $p$ might be also function of inputs, outputs or states of (1a-b). In the latter case the corresponding system is referred to as quasiLPV. The matrix functions in (1a-b), defined as

$$
\left[\begin{array}{c|c}
A(p) & B(p) \\
\hline C(p) & D(p)
\end{array}\right]: \mathbb{P} \rightarrow\left[\begin{array}{l|l}
\mathbb{R}^{n_{\mathrm{x}} \times n_{\mathrm{x}}} & \mathbb{R}^{n_{\mathrm{x}} \times n_{\mathrm{u}}} \\
\hline \mathbb{R}^{n_{\mathrm{y}} \times n_{\mathrm{x}}} & \mathbb{R}^{n_{\mathrm{y}} \times n_{\mathrm{u}}}
\end{array}\right],
$$

are dependent on the instantaneous value of $p$, i.e. $p_{k}=$ $p(k)$, which is called static dependence. Often to simplify (1a-b), it is assumed that $A, B, C, D$ have affine linear static dependence, i.e. they are linear functions of $p=$ $\left[p^{(1)} \ldots p^{\left(n_{\mathrm{p}}\right)}\right]: \quad \star(p)=\star_{0}+\sum_{i=1}^{n_{\mathrm{p}}} \star_{i} p^{(i)}$ where $\star=$ $A, B, C, D$ and $\star_{i}$ is a constant matrix.

Despite the rich history and advances of LPV control synthesis, the system theoretic aspects of statetransformations between LPV-SS representations has not been investigated. Due to this gap in the system theory, researchers have been often led to believe that statetransformations obey the same "rules" as in the LTI case. For example in LPV modeling and identification based on SS models (see Wassink et al. (2004); Lovera and Mercère (2007)), p-dependent state transformations are applied on (1a-b) such as the resulting "equivalent" LPV-SS representation is given by,

$$
\left[\begin{array}{c|c}
T^{-1}\left(p_{k}\right) A\left(p_{k}\right) T\left(p_{k}\right) & T^{-1}\left(p_{k}\right) B\left(p_{k}\right) \\
\hline C\left(p_{k}\right) T\left(p_{k}\right) & D\left(p_{k}\right)
\end{array}\right] .
$$

where $T: \mathbb{P} \rightarrow \mathbb{R}^{n_{\mathrm{x}} \times n_{\mathrm{x}}}$ is a matrix rational function of $p$ invertible for all $p_{k} \in \mathbb{P}$.

However in Tóth (2010) it has been pointed out that this intuitive use of the LTI system theory in the LPV setting can introduce arbitrary large error in the resulting SS form even for slowly varying $p$. As it will be described in Sec. 2, the correct $p$-dependent transformation will lead to related matrices of the form $\hat{A}\left(p_{k}, p_{k+1}\right)=T^{-1}\left(p_{k+1}\right) A\left(p_{k}\right) T\left(p_{k}\right)$. This means that the resulting equivalent LPV-SS form contains matrices which not only depend on $p_{k}$ but on its time-shifted version as well. This dynamic-dependence has been encountered and analyzed in LPV control synthesis (Lee and Dullerud (2006)), system theory (Tóth (2010)) and identification (Tóth et al. (2009)). Using state transformation with dynamic dependence has also been proved to characterize all equivalent LPV-SS representations of a given LPV system (Tóth et al. (2011)). However, many control and identification methods in the LPV literature implicitly build on the assumption of the static dependence. Consequently, there is a need to characterize all equivalent LPV-SS representation with static dependence.

In LPV control synthesis, to avoid the use of dynamic dependence, state-transformation matrices are often re- 
stricted to be constants, i.e. not dependent on $p$. However, as one of the new results of this paper, it is shown in Sec. 3.1 that constant state-transformations are not sufficient to characterize all the classes of equivalent and affine LPV-SS representations. Unlike with parameter independent $T$, there exists a large class of $p$-dependent statetransformations, which by satisfying certain conditions, provide equivalence relation between LPV-SS representations with affine-static dependence. This observation has a primary importance w.r.t. control, filtering, identification or diagnosis of LPV systems, as it provides an additional degree of freedom for synthesis which can be exploited to improve performance.

In this paper we aim to give a detailed analysis of the LPV state-transformation problem. In particular, we investigate the generic structure of the state transformation which characterizes all equivalent LPV-SS representations and investigate when such a transformation preserves staticdependence. The case of affine static dependence and the relating equivalence classes are analyzed in detail and exact conditions with geometric interpretation are given. Furthermore, we also aim to investigate how such statetransformations can be synthesized in practice and what practical relevance they have in terms of identification or control synthesis.

\section{GENERIC STATE TRANSFORMATIONS FOR LPV REPRESENTATIONS}

Consider (1a-b) and introduce a $p$-dependent statetransformation $T(p)$, where $T: \mathbb{P} \rightarrow \mathbb{R}^{n_{\mathrm{x}} \times n_{\mathrm{x}}}$ is a rational matrix function with $\operatorname{det}\left(T\left(p_{k}\right)\right) \neq 0$ and $T\left(p_{k}\right)$ bounded for all $p_{k} \in \mathbb{P}$. Define the new state variable $\hat{x}(k)$ as

$$
x(k)=T\left(p_{k}\right) \hat{x}(k),
$$

for an arbitrary time-sample $k \in \mathbb{Z}$. Note that due to the boundedness and full-rank property of $T\left(p_{k}\right)$ for any $p_{k} \in \mathbb{P}$ and $k \in \mathbb{Z}, T(p)$ corresponds to a bijective mapping. Substituting (4) into (1a-b), gives

$$
q\left(T\left(p_{k}\right) \hat{x}(k)\right)=A\left(p_{k}\right) T\left(p_{k}\right) \hat{x}(k)+B\left(p_{k}\right) u(k),
$$

where $q$ is the forward time-shift operator, i.e. $q x(k)=$ $x(k+1)$. As $q T\left(p_{k}\right)=T\left(p_{k+1}\right)$, i.e. multiplication by $q$ is not commutative over a $p$-dependent coefficient, (5) is equivalent with

$$
\begin{aligned}
\hat{x}(k+1)=T^{-1}\left(p_{k+1}\right) A\left(p_{k}\right) & T\left(p_{k}\right) \hat{x}(k) \\
& +T^{-1}\left(p_{k+1}\right) B\left(p_{k}\right) u(k) .
\end{aligned}
$$

As (6) is a first-order difference equation with $\hat{x}$ being in equivalence relation with $x$, thus (6) qualifies as an equivalent SS representation in the form:

$$
\left[\begin{array}{c|c}
T^{-1}\left(p_{k+1}\right) A\left(p_{k}\right) T\left(p_{k}\right) & T^{-1}\left(p_{k+1}\right) B\left(p_{k}\right) \\
\hline C\left(p_{k}\right) T\left(p_{k}\right) & D\left(p_{k}\right)
\end{array}\right] .
$$

The resulting state-space matrices depend on the future value of the scheduling variable $p$, which is a particular non-causal dynamic dependence. Such a dynamic dependence is not only an "artifact" of the state transformation but can also be encountered in the state-space realization problem of LPV-IO representations (Tóth et al. (2011)) and in modeling of general nonlinear systems in an LPV form (Tóth (2010)) or in the case of unknown input reconstruction (Kulcsár et al. (2009)). Next, we investigate how we can reformulate the concept of SS representations to handle dynamic dependence in a well-founded sense and how we can characterize equivalent representations. For this purpose the algebraic framework of the LPV behavioral approach (Tóth et al. (2011)) is briefly introduced.

2.1 Equivalent state-space representation with dynamic scheduling dependence

A general form of LPV-SS representations can be formulated as a first-order parameter-varying difference equation system interpreted as a first-order polynomial in the timeoperator $q$. This allows to characterize equivalent differential equations and specify when solution sets are equal by an algebraic approach. To define the possibly dynamic functional dependence of a single coefficient, i.e. element of a matrix, in such a representation, we employ functions belonging to the field $\mathcal{R}=\cup_{n \in \mathbb{N}} \mathcal{R}_{n}$, where $\mathcal{R}_{n}$ is the set of real-meromorphic functions $r: \mathbb{R}^{n} \rightarrow \mathbb{R}$ where $r\left(\mathrm{x}_{1}, \cdots, \mathrm{x}_{n}\right)$ does depend on $\mathrm{x}_{n}$. Then the general LPVSS representation is defined as

$$
\begin{aligned}
x(k+1) & =(A \diamond p)(k) x(k)+(B \diamond p)(k) u(k), \\
y(k) & =(C \diamond p)(k) x(k)+(D \diamond p)(k) u(k),
\end{aligned}
$$

with

$$
\left[\begin{array}{c|c}
A & B \\
\hline C & D
\end{array}\right] \in\left[\begin{array}{l|l}
\mathcal{R}^{n_{\mathrm{x}} \times n_{\mathrm{x}}} & \mathcal{R}^{n_{\mathrm{x}} \times n_{\mathrm{u}}} \\
\hline \mathcal{R}^{n_{\mathrm{y}} \times n_{\mathrm{x}}} & \mathcal{R}^{n_{\mathrm{y}} \times n_{\mathrm{u}}}
\end{array}\right] .
$$

Here the operator $\diamond:\left(\mathcal{R}, \mathbb{P}^{\mathbb{Z}}\right) \rightarrow \mathbb{R}^{\mathbb{Z}}$ is defined by $r \diamond$ $p=r\left(p, q p, q^{-1} p, \ldots\right)$, where $\mathbb{X}^{\mathbb{Z}}$ stands for all maps from $\mathbb{Z}$ to $\mathbb{X}$. Thus the value of a ( $p$-dependent) coefficient $r$ in an LPV system representation at time $k$ is given by $(r \diamond$ $p)(k)$. Furthermore, the latent variable $x$ in (8a) naturally fulfills the property of a state variable.

Now we can consider the state transformation (5) in this setting. Let $T \in \mathcal{R}^{n_{\mathrm{x}} \times n_{\mathrm{x}}}$ be invertible (in $\mathcal{R}^{n_{\mathrm{x}} \times n_{\mathrm{x}}}$ ), then $x=(T \diamond p) \hat{x}$ implies that

$$
q(T \diamond p) \hat{x}=(A \diamond p)(T \diamond p) \hat{x}+(B \diamond p) u .
$$

Due to the fact that $(10)$ is a first-order parameter varying difference equation w.r.t. $\hat{x}$ and $T \in \mathcal{R}^{n_{\mathrm{x}} \times n_{\mathrm{x}}}$ is invertible, $\hat{x}$ qualifies as a new state variable which yields an equivalent LPV-SS representation of (8a-b). This implies the following definition:

Definition 1. (Equivalence). Consider two LPV-SS representations, with $\mathrm{SS}$ matrices $(A, B, C, D)$ and $(\hat{A}, \hat{B}, \hat{C}, \hat{D})$ in $\mathcal{R}^{\cdot \times \cdot}$ where $A \in \mathcal{R}^{n_{1} \times n_{1}}$ and $\hat{A} \in \mathcal{R}^{n_{2} \times n_{2}}$ and $n_{1} \geq n_{2}$. For a given scheduling dimension $n_{\mathrm{p}}$, these representations are called equivalent,

$$
\left[\begin{array}{c|c}
A & B \\
\hline C & D
\end{array}\right] \stackrel{n_{\mathrm{p}}}{\sim}\left[\begin{array}{c|c}
\hat{A} & \hat{B} \\
\hline \hat{C} & \hat{D}
\end{array}\right],
$$

if there exists an invertible $T \in \mathcal{R}^{n_{1} \times n_{1}}$ such that:

$$
\begin{aligned}
\vec{T} A T^{-1} & =\left[\begin{array}{ll}
\hat{A} & 0 \\
* & *
\end{array}\right], \vec{T} B=\left[\begin{array}{l}
\hat{B} \\
*
\end{array}\right], \begin{array}{ll}
\uparrow & n_{2} \\
& n_{1}-n_{2}
\end{array} \\
C T^{-1} & =\left[\begin{array}{ll}
\hat{C} & 0
\end{array}\right], \quad D_{1}=D_{2},
\end{aligned}
$$

where $\vec{T} \diamond p=T \diamond(q p)$. In case $n_{2}>n_{1}$ definition of (12) follows respectively.

Similar to the LTI case it can be proved that two LPV state-space representations have the same IO behavior (projected solution set of $(8 \mathrm{a}-\mathrm{b})$ w.r.t. $(u, y))$ in an almosteverywhere sense if and only if their state variables are related via a state-transformation:

$$
(T \diamond p)(k) \hat{x}(k)=\left[\begin{array}{clll}
x(k) & & \uparrow & n_{2} \\
* & \uparrow & & n_{1}-n_{2}
\end{array} \quad \forall k \in \mathbb{Z} .\right.
$$


For a proof see Tóth (2010). Note that the IO behaviors are almost-everywhere equal due to the fact that $T$ is meromorphich, hence it is bounded only almost everywhere w.r.t. $\mathbb{P}^{\mathbb{Z}}$. Using the concept of SS equivalence relation the definition of LPV-SS state-minimality, equivalence classes and canonical forms follows naturally (see Tóth (2010)).

\subsection{Computation of generic state transformations}

The generic state-transformation, proposed in the previous section, provides an equivalence transformation between a large class of LPV-SS representations with dynamic dependence. However it becomes a question how to compute such a state-transformation if two LPV-SS representations are given. Or in other words, how can we verify that they belong to the same equivalence-class or not?

Consider two LPV-SS representations with SS matrices $\left(A_{1}, B_{1}, C_{1}, D_{1}\right)$ and $\left(A_{2}, B_{2}, C_{2}, D_{2}\right)$ in $\mathcal{R} \cdot \times \cdot$ where $A_{1} \in$ $\mathcal{R}^{n_{1} \times n_{1}}$ and $A_{2} \in \mathcal{R}^{n_{2} \times n_{2}}$ and $n_{1}>n_{2}$. In terms of Def. (1), these representations are equivalent if there exist a $M_{1} \in \mathcal{R}^{n_{1} \times n_{2}}$ and a $M_{2} \in \mathcal{R}^{n_{2} \times n_{1}}$ such that

$$
\begin{array}{ll}
A_{1} \diamond p=\left(M_{1} A_{2} M_{2}\right) \diamond p, & B_{1} \diamond p=\left(M_{1} B\right) \diamond p, \\
C_{1} \diamond p=\left(C_{2} M_{2}\right) \diamond p, & I_{n_{2} \times n_{2}}=\left(M_{2} \overleftarrow{M}_{1}\right) \diamond p
\end{array}
$$

where $\leftarrow$ męans backward time-shift in the coefficient dependence: $\overleftarrow{M}_{1} \diamond p=M_{1} \diamond\left(q^{-1} p\right)$. The conditions (14ab) imply the existence of a state-transformation $T=$ $\left[M_{2}^{\top} *\right]^{\top}$ such that the two SS representation are equivalent. Furthermore, (14a-b) corresponds to a system of bi-linear equations meaning that it is an algebraic problem to find the state transformation matrix between two given representations. If no solution exist it means that the two representations are not equivalent. However, the coefficients in this algebraic problem are not constant values but functions, which means that a solution can be computed only via a symbolic solver. In general, the symbolic solution of the state transformation problem is a computationally demanding operation with exponentially increasing memory load. Therefore, in terms of practical use, computing the state transformation via (14a) is limited to small scale problems and can not be used to assist the existing control synthesis schemes.

An alternative way of computation of the state-transformation matrix follows through the so called $n$-step observability reachability matrices:

Definition 2. (based on Gohberg et al. (1992)). In discrete time, the $n$-step state-observability matrix function $\mathcal{O}_{n} \in$ $\mathcal{R}^{\left(n n_{\mathrm{y}}\right) \times n_{\mathrm{x}}}$ and state-reachability matrix function $\mathcal{R}_{n} \in$ $\mathcal{R}^{n_{\mathrm{x}} \times\left(n n_{\mathrm{u}}\right)}$ of a given LPV-SS representation with SS matrices $(A, B, C, D)$ and state, input and output dimensions $n_{\mathrm{x}}, n_{\mathrm{u}}$ and $n_{\mathrm{y}}$ respectively is defined as

$$
\begin{array}{llll}
\mathcal{O}_{n}=\left[\begin{array}{lllll}
\mathrm{o}_{1}^{\top} & \mathrm{o}_{2}^{\top} & \ldots & \mathrm{o}_{n}^{\top}
\end{array}\right]^{\top}, \quad \mathcal{R}_{n}=\left[\begin{array}{llll}
\mathrm{r}_{1} & \mathrm{r}_{2} & \ldots & \mathrm{r}_{n}
\end{array}\right], \\
\text { where } \\
\mathrm{o}_{1}=C, \quad \mathrm{o}_{i+1}=\overrightarrow{\mathrm{o}_{i}} A, \quad \forall i>1, \\
\mathrm{r}_{1}=B, & \mathrm{r}_{i+1}=A \overleftarrow{\mathrm{r}_{i}}, \quad \forall i>1 .
\end{array}
$$

It can be shown that boundedness and full rank of $\mathcal{O}_{n_{\mathrm{x}}}$ (or $\mathcal{R}_{n_{\mathrm{x}}}$ ) for every $\mathbb{P}^{\mathbb{Z}}$ are sufficient, but not necessary conditions for the complete state observability (reachability) of a given LPV-SS representation with state dimension $n_{\mathrm{x}}$ (Silverman and Meadows (1967)). Full rank property (in $\mathcal{R} \cdot \times \cdot$ ) of $\mathcal{O}_{n_{\mathrm{x}}}\left(\right.$ or $\left.\mathcal{R}_{n_{\mathrm{x}}}\right)$ guarantees state observability (reachability) in a weaker sense which is called structural state-observability. Nevertheless the following theorem explicitly states the effect of a state-transformation on these matrices:

Theorem 1. (based on Silverman and Meadows (1965)). If the matrices of two LPV-SS representations, with state dimensions $n_{\mathrm{x}}$ and with a common $n_{\mathrm{p}}$ dimensional scheduling space, fulfill the equivalence relation in terms of Def. 1 via the state-transformation $T \in \mathcal{R}^{n_{\mathrm{x}} \times n_{\mathrm{x}}}$, then for all $n \in \mathbb{N}$ :

$$
\hat{\mathcal{O}}_{n}=\mathcal{O}_{n} T \text { and } \quad \hat{\mathcal{R}}_{n}=\vec{T}^{-1} \mathcal{R}_{n}
$$

hold, where $\mathcal{O}_{n}$ and $\hat{\mathcal{O}}_{n}$, respectively $\mathcal{R}_{n}$ and $\hat{\mathcal{R}}_{n}$, are the corresponding $n$-step state-observability/reachability matrices of the representations.

The proof of this theorem similarly follows as in Silverman and Meadows (1965). Now we can use this theorem to provide a new and simple computation of the state transformation between two given LPV-SS representations if it holds true that their corresponding $n_{\mathrm{x}}$-step stateobservability or state-reachability matrices are full rank. Using (16) and by choosing $n=n_{\mathrm{x}}$ it follows that

$$
T=\mathcal{O}_{n_{\mathrm{x}}}^{-\ell} \cdot \hat{\mathcal{O}}_{n_{\mathrm{x}}} \text { and } \quad \vec{T}^{-1}=\hat{\mathcal{R}}_{n_{\mathrm{x}}} \cdot \mathcal{R}_{n_{\mathrm{x}}}^{-r},
$$

under the condition that $\mathcal{O}_{n_{\mathrm{x}}}, \hat{\mathcal{O}}_{n_{\mathrm{x}}}, \mathcal{R}_{n_{\mathrm{x}}}$ and $\hat{\mathcal{R}}_{n_{\mathrm{x}}}$ are all full rank, moreover $\star^{-\ell,-r}$ denote the pseudo left and right inverse. Note that each of these formulas can be used to compute $T$ however the observability matrix based solution has far less computational need than the reachability matrix based solution. These formulas can be easily implemented and do not need any symbolic solver as in the previous case.

\section{PRESERVING STATIC DEPENDENCE VIA SIMILARITY STATE TRANSFORMATIONS}

Previously we have seen that with the proposed generic state transformation (13) we can establish equivalence relation for the class of LPV-SS representations with dynamic dependence. However, most of the available control synthesis approaches and state-space identification methods in the LPV setting implicitly build upon SS representation forms including only static dependence. In the static case, is it enough to consider only constant state transformations to characterize LPV-SS equivalence?

Consider the set of static LPV-SS representations, where the scheduling dependence can be written as a static affine linear dependence, i.e. the parameter varying matrices can be described as a linear combination of coefficient matrices weighted by component-wise scheduling parameters:

$$
\begin{aligned}
x(k+1) & =\sum_{i=0}^{n_{\mathrm{p}}} p_{k}^{(i)}\left(A^{(i)} x(k)+B^{(i)} u(k)\right), \\
y(k) & =\sum_{i=0}^{n_{\mathrm{p}}} p_{k}^{(i)}\left(C^{(i)} x(k)+D^{(i)} u(k)\right),
\end{aligned}
$$

where $p_{k}=\left[p_{k}^{(1)} \ldots p_{k}^{\left(n_{\mathrm{p}}\right)}\right] \in \mathbb{R}^{n_{\mathrm{p}}}$ is the scheduling vector and $p_{k}^{(0)} \equiv 1$. In the above discrete-time, affine LPV description, $\star^{(i)}$ with $\star=A, B, C, D$ denote real valued coefficient matrices with appropriate dimensions based on the signal spaces $n_{\mathrm{x}}, n_{\mathrm{u}}$ and $n_{\mathrm{y}}$ respectively. Note that (18a-b) can be also considered in the form where the state-space 
matrices are linear combinations of linearly independent functions of $p$. As we will see the upcoming results also trivially hold in that case. Furthermore, to simplify the discussion we consider equivalence relations only between equidimensional LPV-SS representations $\left(n_{\mathrm{x}}=n_{\hat{\mathrm{x}}}\right)$. The following theorem characterizes the conditions the that state transformation $(T \diamond p)$ must fulfill to preserve static affine dependence in this case.

Theorem 2. Given a set of equivalent discrete time, equidimensional LPV-SS representations with affine linear static $p$-dependence. All representations of this set are characterized via parameter-dependent state transformations $(T \diamond p)$ satisfying conditions in (20), (21) and (25).

Proof 1. Consider a LPV-SS representation in the form of $(18 \mathrm{a}-\mathrm{b})$ and a generic parameter-varying similarity transformation $x(k)=(T \diamond p)(k) \hat{x}(k)$ implying $(10)$. Then in terms of $(12)$ :

$$
\begin{aligned}
((T \diamond p)(k+1))^{-1} \sum_{i=0}^{n_{\mathrm{p}}} p_{k}^{(i)} A^{(i)}(T \diamond p)(k) & =\sum_{i=0}^{n_{\mathrm{p}}} p_{k}^{(i)} \hat{A}^{(i)}, \\
((T \diamond p)(k+1))^{-1} \sum_{i=0}^{n_{\mathrm{p}}} p_{k}^{(i)} B^{(i)} & =\sum_{i=0}^{n_{\mathrm{p}}} p_{k}^{(i)} \hat{B}^{(i)}, \\
\sum_{i=0}^{n_{\mathrm{p}}} p_{k}^{(i)} C^{(i)}(T \diamond p)(k) & =\sum_{i=0}^{n_{\mathrm{p}}} p_{k}^{(i)} \hat{C}^{(i)} .
\end{aligned}
$$

Assume that $(T \diamond p)(k)$ is invertible for all possible scheduling trajectories of the system at anytime, i.e. for all $p \in \mathbb{P}^{\mathbb{Z}}$ and $k \in \mathbb{Z}$. The left-hand side of $(19 \mathrm{~b})$ implies that premultiplication of $\sum_{i=0}^{n_{\mathrm{p}}} p_{k}^{(i)} B^{(i)}$ by $((T \diamond p)(k+1))^{-1}$ needs to result in only static affine dependence. Therefore

$(T \diamond p)(k+1) \hat{B}^{(i)} p_{k}^{(i)}=(\hat{Z} \diamond p)^{(i)}(k, k+1)+\sum_{j=0}^{n_{\mathrm{p}}} p_{k}^{(j)} Z^{(i, j)}$

where

$$
\sum_{i=0}^{n_{\mathrm{p}}}(\hat{Z} \diamond p)^{(i)}(k, k+1)=0 .
$$

Similarly, (19c) can be written as

$$
p_{k}^{(i)} C^{(i)}(T \diamond p)(k)=(\hat{W} \diamond p)^{(i)}(k)+\sum_{j=0}^{n_{\mathrm{p}}} p_{k}^{(j)} W^{(i, j)}
$$

where

$$
\sum_{i=0}^{n_{\mathrm{p}}}(\hat{W} \diamond p)^{(i)}(k)=0 .
$$

On the other hand, pre-multiplying both sides of eq. (19a) by $(T \diamond p)(k+1)$ leads to,

$$
\sum_{i=0}^{n_{\mathrm{p}}} p_{k}^{(i)} A^{(i)}(T \diamond p)(k)=(T \diamond p)(k+1) \sum_{i=0}^{n_{\mathrm{p}}} p_{k}^{(i)} \hat{A}^{(i)} .
$$

The right-hand side can be rewritten as $(T \diamond p)(k+$ 1) $\hat{A}^{(i)} p_{k}^{(i)}=(\hat{X} \diamond p)^{(i)}(k, k+1)+\sum_{j=0}^{n_{\mathrm{p}}} p_{k}^{(j)} X^{(i, j)}$. Therefore,

$$
\begin{aligned}
\sum_{i=0}^{n_{\mathrm{p}}} p_{k}^{(i)} & A^{(i)}(T \diamond p)(k)= \\
& \sum_{i=0}^{n_{\mathrm{p}}}\left((\hat{X} \diamond p)^{(i)}(k, k+1)+\sum_{j=0}^{n_{\mathrm{p}}} p_{k}^{(j)} X^{(i, j)}\right) .
\end{aligned}
$$

On the other hand,

$$
\begin{aligned}
p_{k}^{(i)} A^{(i)}(T \diamond p)(k) & =\sum_{j=0}^{n_{\mathrm{p}}} p_{k}^{(j)} Y^{(i, j)}+(\hat{Y} \diamond p)^{(i)}(k) \quad(24) \\
\sum_{i=0}^{n_{\mathrm{p}}} p_{k}^{(i)} A^{(i)}(T \diamond p)(k) & =\sum_{i=0}^{n_{\mathrm{p}}}\left(\sum_{j=0}^{n_{\mathrm{p}}} p_{k}^{(j)} Y^{(i, j)}+(\hat{Y} \diamond p)^{(i)}(k)\right)
\end{aligned}
$$

which imply that the following condition has to be fulfilled

$$
\sum_{i=0}^{n_{\mathrm{p}}}\left((\hat{Y} \diamond p)^{(i)}(k)-(\hat{X} \diamond p)^{(i)}(k, k+1)\right)=0 .
$$

Consequently, a dynamic parameter dependent similarity state transformation can be used to connect equivalent affine, discrete-time LPV-SS state space representations if conditions (20), (21) and (25) are met.

The conditions in Th. 2 clearly show that characterization of all equivalent LPV-SS classes, even with linear affine and static dependence, require similarity state transformation with dynamic scheduling parameter dependence, which is a major conclusion.

In the past it has been been assumed that similarity state transformations between LPV-SS representations with linear affine and static dependence are completely characterized by a constant state transformation matrix, $T^{(0)}$, resulting in the following transformation rules

$$
\begin{aligned}
\left(T^{(0)}\right)^{-1} A^{(i)} T^{(0)} & =\hat{A}^{(i)} & \left(T^{(0)}\right)^{-1} B^{(i)} & =\hat{B}^{(i)} \\
C^{(i)} T^{(0)} & =\hat{C}^{(i)} & D^{(i)} & =\hat{D}^{(i)}
\end{aligned}
$$

with $i=0, \ldots n_{\mathrm{p}}$. This constant $T^{(0)}$ projects the appropriate affine term onto its equivalent component. Accordingly, no cross-terms are present in between affine coefficient components. One can observe in (23) and (24) that terms $X^{(i, j)}$ and $Y^{(i, j)}$ for all $i, j=0 \ldots n_{\mathrm{p}}$ precisely characterize cross term relations in between affine SS matrices. Comparing therefore the results of Th. 2 with the 'time invariant" relation in (26a-b), conservativeness of the latter is salient. In addition to, results in Th. 2 shows how, by using parameter dependent state transformation matrix, the information in coefficients $A^{(i)}$ with $i$ fixed can be dispersed to $\hat{A}^{(j)} \forall j=0, \ldots n_{\mathrm{p}}$ which indeed is not possible in $(26 \mathrm{a}-\mathrm{b})$. It is important to note that even if symbolic or observability/reachability matrix based calculation of $T$ to characterize similarity transformation between LPV-SS representations is possible, in the current synthesis tools affine static dependence of the matrices and also the transformations is a core assumption. Hence it is important to investigate how the conditions on the equivalnce transformation changes if we restrict $T$ to have static linear affine dependence on $p$, i.e. $T\left(p_{k}\right)=\sum_{i=0}^{n_{\mathrm{p}}} p_{k}^{(i)} T^{(i)}$.

3.1 Affine parameter dependent state transformation with static dependence

In the sequel we will investigate how an affine state transformation matrix can be used to connect affine LPVSS representations by using static parameter dependence. Theorem 3. Given two equivalent discrete time, equidimensional LPV-SS representations with affine linear static $p$-dependence. Let $x(k)=T\left(p_{k}\right) \hat{x}(k)$ be an invertible state-transformation for all $p_{k} \in \mathbb{P}$ where $T\left(p_{k}\right)=$ $\sum_{i=0}^{n_{\mathrm{p}}} T^{(i)} p_{k}^{(i)}$. The two representations are equivalent in terms of Def. 1 if there exists a transformation matrix satisfying conditions (27b), (27c) and (29). 
Proof 2. Using $T\left(p_{k+1}\right)=\sum_{i=0}^{n_{\mathrm{p}}} T^{(i)} p_{k+1}^{(i)}$, (19a-c) read as

$$
\begin{aligned}
\sum_{i=0}^{n_{\mathrm{p}}} T^{(i)} p_{k+1}^{(i)} \sum_{j=0}^{n_{\mathrm{p}}} \hat{A}^{(j)} p_{k}^{(j)} & =\sum_{j=0}^{n_{\mathrm{p}}} A^{(j)} p_{k}^{(j)} \sum_{i=0}^{n_{\mathrm{p}}} T^{(i)} p_{k}^{(i)} \\
\sum_{i=0}^{n_{\mathrm{p}}} T^{(i)} p_{k+1}^{(i)} \sum_{j=0}^{n_{\mathrm{p}}} \hat{B}^{(j)} p_{k}^{(j)} & =\sum_{j=0}^{n_{\mathrm{p}}} B^{(j)} p_{k}^{(i)} \\
\sum_{j=0}^{n_{\mathrm{p}}} C^{(j)} p_{k}^{(j)} \sum_{i=0}^{n_{\mathrm{p}}} T^{(i)} p_{k}^{(i)} & =\sum_{j=0}^{n_{\mathrm{p}}} \hat{C}^{(j)} p_{k}^{(i)} .
\end{aligned}
$$

Evaluating separately both sides of (27a) gives

$$
\begin{aligned}
\sum_{i=0}^{n_{\mathrm{p}}} T^{(i)} p_{k+1}^{(i)} \sum_{j=0}^{n_{\mathrm{p}}} \hat{A}^{(j)} p_{k}^{(j)} & =\sum_{i=0}^{n_{\mathrm{p}}} X^{(i)} p_{k}^{(i)}+\hat{X}\left(p_{k}, p_{k+1}\right) \\
\sum_{j=0}^{n_{\mathrm{p}}} A^{(j)} p_{k}^{(j)} \sum_{i=0}^{n_{\mathrm{p}}} T^{(i)} p_{k}^{(i)} & =\sum_{i=0}^{n_{\mathrm{p}}} Y^{(i)} p_{k}^{(i)}+\hat{Y}\left(p_{k}\right),
\end{aligned}
$$

where the condition of existence for an affine statetransformation is

$$
\hat{Y}\left(p_{k}\right)-\hat{X}\left(p_{k}, p_{k+1}\right)=0 \text {. }
$$

Condition (29) is a (polynomial) matrix difference equation.

\section{COMPUTATIONAL ISSUES}

Next we demonstrate on a simple example the importance of $p$-dependent state-transformation and through of this example we also give alternative interpretations for conditions (27a-c) and (29).

Example 1. For the sake of simplicity consider $n_{\mathrm{p}}=2$. Our aim is to develop conditions under which $T\left(p_{k}\right)$ exists with linear affine static dependence to connect two SS representations $(A, B, C, D)$ and $(\hat{A}, \hat{B}, \hat{C}, \hat{D})$ of an LPV system, where each matrix has linear affine static $p$ dependence, and compare the results with constant statetransformation. Assume that here is an invertible (square) transformation matrix $T\left(p_{k}\right)=T^{(0)}+T^{(1)} p_{k}^{(1)}$ for all $j \in \mathbb{Z}$ such that $(A, B, C, D)$ and $(\hat{A}, \hat{B}, \hat{C}, \hat{D})$ satisfy the equivalence relation. If condition

$$
T^{(1)} p_{k+1}^{(1)}\left(\hat{A}^{(0)}+\hat{A}^{(1)} p_{k}^{(1)}\right)-A^{(1)} T^{(1)}\left(p_{k}^{(1)}\right)^{2}=0,
$$

is satisfied, we can write,

$$
\begin{aligned}
& T^{(0)} \hat{A}^{(0)}+T^{(0)} \hat{A}^{(1)} p_{k}^{(1)}= \\
& A^{(0)} T^{(0)}+p_{k}^{(1)}\left(A^{(0)} T^{(1)}+A^{(1)} T^{(0)}\right) .
\end{aligned}
$$

This implies that

$$
\begin{aligned}
T^{(0)} \hat{A}^{(0)} & =A^{(0)} T^{(0)} \\
\hat{A}^{(0)} & =\left(T^{(0)}\right)^{-1} A^{(0)} T^{(0)},
\end{aligned}
$$

where $T^{(0)}$ has to be invertible and

$$
\begin{aligned}
T^{(0)} \hat{A}^{(1)} & =\left(A^{(0)} T^{(1)}+A^{(1)} T^{(0)}\right), \\
\hat{A}^{(1)} & =\left(T^{(0)}\right)^{-1}\left(A^{(0)} T^{(1)}+A^{(1)} T^{(0)}\right) .
\end{aligned}
$$

Further conditions on the input and output map transformations are given by

$$
\begin{aligned}
C^{(1)} T^{(1)}\left(p_{k}^{(1)}\right)^{2} & =0, \\
T^{(1)} p_{k+1}^{(1)} \hat{B}^{(0)}+T^{(1)} p_{k+1}^{(1)} \hat{B}^{(1)} p_{k}^{(1)} & =0 .
\end{aligned}
$$

Transformation on the output map boils down to

$$
\begin{aligned}
& \hat{C}^{(0)}=C^{(0)} T^{(0)}, \\
& \hat{C}^{(1)}=C^{(0)} T^{(1)}+C^{(1)} T^{(0)},
\end{aligned}
$$

and finally the transformed input direction reads as

$$
\begin{aligned}
& T^{(0)} \hat{B}^{(0)}=B^{(0)}, \\
& T^{(0)} \hat{B}^{(1)}=B^{(1)} .
\end{aligned}
$$

By only applying an invertible and constant similarity transformation $T$, the transformed SS matrices read as,

$$
\begin{array}{ll}
\hat{A}_{T}^{(0)}=T^{-1} A^{(0)} T, & \hat{A}_{T}^{(1)}=T^{-1} A^{(1)} T, \\
\hat{B}_{T}^{(0)}=T^{-1} B^{(0)}, & \hat{B}_{T}^{(1)}=T^{-1} B^{(1)}, \\
\hat{C}_{T}^{(0)}=C^{(0)} T, & \hat{C}_{T}^{(1)}=C^{(1)} T,
\end{array}
$$

where subscript $\star_{T}$ refers to the constant transformation. Structurally, $A^{(0)}, C^{(0)}$ and $B^{(i)}$ with $i=0,1$ can be related in a similar way as in the constant transformation case, however, $A^{(1)}, C^{(1)}$ are projected differently. This clearly shows that a $p$-dependent state transformation represents an additional degree of freedom and hence constant state transformations cannot characterize all equivalent SS representations, with affine linear and static $p$-dependence, of the same LPV system even in this simple case. Note also, invertibility of $T^{(0)}$ is a necessary condition for the existence of $T\left(p_{k}\right)$.

Difference-algebraic interpretation. Eq. (30) and (34a) are difference-algebraic conditions where time variation is encapsulated into $p_{k}$ and $p_{k+1}$. Consequently, if the one-step-ahead scheduling variable together with the actual scheduling value satisfies the difference equality constraints for all $k \in \mathbb{Z}$, the state-transformation preserves affinity in transforming $A^{(i)}$ and $C^{(i)}$ to $\hat{A}^{(i)}$ and $\hat{C}^{(i)}$, $i=0,1$. Based on this, the scheduling variable has to follow a (set of) trajectories described by (30)-(34b). From differential-algebraic perspective, however, this might be a clear limitation of the state-transformation since $p_{k}$ is usually an arbitrary but time-varying exogenous or endogenous (quasi LPV systems) signal. If the scheduling parameters are originated from an exogenous source, then we can only use a posteori test to check conditions (30)(34b). If scheduling vector is derived from endogenous variables and we intend to use affine transformation matrix structure, the above mentioned conditions become dynamic equality constraints to be respected. Henceforward, the entire parameter trajectory has to be restricted and the validity domain of the transformed SS model might reduce intensively if it exist at all. Or conversely, these conditions can also naturally be ensured by the system's dynamics itself.

Geometric interpretation. Instead of satisfying equality conditions in (30), (34a) and (34b) (or generally speaking in $(27 \mathrm{~b}),(27 \mathrm{c})$ and (29)), we can directly apply component-wise matrix algebraic conditions. From this point of view, geometrical concepts play an important role to interpret the following results. As a direct consequence, we can write,

$$
\begin{array}{ll}
T^{(1)} \hat{B}^{(0)}=0, & T^{(1)} \hat{B}^{(1)}=0, \\
T^{(1)} \hat{A}^{(0)}=0, & T^{(1)} \hat{A}^{(1)}=0, \\
A^{(1)} T^{(1)}=0 & C^{(1)} T^{(1)}=0 .
\end{array}
$$


In the above equations, it is claimed that $T^{(1)}$ has to be a left zero divisor of $\hat{B}^{(i)}, \hat{A}^{(i)}, i=0,1$ and right zero divisor of $A^{(1)}$ and $C^{(1)}$. Based on this property, $T^{(1)}$ is not invertible. Denote the affine output mapping by $\mathcal{C}^{(i)}$, $i=0,1$ and by $\mathcal{T}^{(i)}, i=0,1$ the affine state transformation (mapping). Geometric conditions guaranteeing the existence of the affine state-transformation matrix at the plant output can now be given as,

$$
\operatorname{Im}\left(\mathcal{T}^{(1)}\right) \subseteq \operatorname{Ker}\left(\mathcal{C}^{(1)}\right)
$$

where the image of state-to-state similarity mapping has to be contained in the kernel of the output projection whose matrix representation is $C^{(1)}$. In addition to, images of the transformed input mapping $\mathcal{B}^{(0)}$ and $\mathcal{B}^{(1)}$ have to be contained in the complementary image space of $\mathcal{T}^{(1)}$ w.r.t to the entire state-space, i.e.

$$
\operatorname{Im}\left(\mathcal{B}^{(i)}\right) \subseteq \operatorname{Im}\left(\mathcal{T}^{(1)}\right)^{\perp}, \quad \text { for } i=0,1 .
$$

Finally, conditions on $A$, see (38b-c) (or (30)), can be also characterized in the geometric framework. Accordingly, images of the state-to-state mapping of the transformed system denoted by $\mathcal{A}^{(i)}, i=0,1$ has to live in $\operatorname{Im}\left(\mathcal{T}^{(1)}\right)^{\perp}$. Let us generalize conditions (38a)-(38c) to an $n_{p}$ dimensional LPV-SS representations. Define the set of equality constraints, conditions by,

$$
\mathcal{L}=0 \Leftrightarrow \mathcal{L}_{B}=0, \quad \mathcal{L}_{A}=0, \quad \mathcal{L}_{C}=0, \quad \exists\left(T^{(0)}\right)^{-1}
$$

where $\mathcal{L}_{\star}$ denotes the set of equality constraints derived from $(27 b),(27 c)$ and (29). This type of interpretations is a component-wise matrix solution to satisfy the conditions of existence for affine $T\left(p_{k}\right)$ regardless to the scheduling trajectory.

Numerical example. Let us consider the following $n_{p}=$ $n_{X}=2$ SISO example by,

$$
\begin{gathered}
A^{(1)}=\left[\begin{array}{cc}
-.05 & .05 \\
.1 & -.1
\end{array}\right], \quad A^{(2)}=\left[\begin{array}{ll}
0 & .1 \\
0 & -.2
\end{array}\right] \\
{\left[B^{(1)} B^{(2)}\right]=\left[\begin{array}{cc}
-.05 & .2 \\
1 & -.4
\end{array}\right], \quad\left[C^{(1)^{T}} C^{(2)^{T}}\right]=\left[\begin{array}{ll}
1 & .0 \\
1 & 1
\end{array}\right] .}
\end{gathered}
$$

By applying the parameter varying similarity state transformation as,

$$
T^{(1)}=\left[\begin{array}{cc}
1 & 0 \\
-1 & 1
\end{array}\right], T^{(2)}=\alpha \cdot\left[\begin{array}{ll}
1 & 1 \\
0 & 0
\end{array}\right],
$$

where $\alpha$ is a finite scalar, componentwise conditions (38ac) are satisfied. Note, difference-algebraic conditions required for the existence of affine parameter dependent SS transformation also hold. If $\alpha=0$, the state transformation problem results in a parameter independent solution. Considering the case $\alpha=0$ as the constant transformation problem and $\alpha \neq 0$ as the affine $p$-dependent solution, we can conclude the freedom in both $\hat{A}_{\alpha \neq 0}^{(2)}$ and $\hat{C}_{\alpha \neq 0}^{(2)}$ since all other coefficient matrices are identical (e.g. $\hat{A}_{\alpha=0}^{(1)}=$ $\left.\hat{A}_{\alpha \neq 0}^{(1)}\right)$. In this specific case of example, one can exploit the degree of freedom denoted by $\alpha$ in e.g. state observer design.

\section{CONCLUSION}

In this paper, we have investigated the problem of generic similarity state-transformations for LPV state-space representations. As a major result it has been shown that equivalnce classes of representations with static dependence are not characterized by constant state transformations. Conditions on state-transformation with dynamic dependence has been derived which ensure preservation of static dependence of the original SS representation via the state-transformation. In case of affine LPV representations constructive conditions have been derived allowing numerical computation of the transformation. The achieved results opens up new possibilities in LPV-SS identification, control or system diagnosis due to the additional degree of freedom that can be exploited in terms of statetransformations.

\section{REFERENCES}

Apkarian, P. and Gahinet, P. (1995). A convex characterization of gain-scheduled $\mathcal{H}_{\infty}$ controllers. IEEE Trans. on Automatic Control, 40(5), 853-864.

Felici, F., van Wingerden, J.W., and Verhaegen, M. (2006). Subspace identification of MIMO LPV systems using a periodic scheduling sequence. Automatica, 43(10), 16841697.

Gohberg, I., Kaashoek, M.A., and Lerer, L. (1992). Minimality and realization of discrete time-varying systems. In: Time-variant systems and interpolation, I. Gohberg, Ed., 261-296. Birkhäuser Verlag.

Kulcsár, B., Bokor, J., and Shinar, J. (2009). Unknown input reconstruction for LPV systems. International Journal of Robust and Nonlinear Control, 20(5), 579595.

Lee, J.W. and Dullerud, G.E. (2006). Optimal disturbance attenuation for discrete-time switched and Markovian jump linear systems. SIAM Journal on Control and Optimization, 45(4), 1329-1358.

Lee, L.H. and Poolla, K.R. (1996). Identification of linear parameter-varying systems via LFTs. In Proc. of the 35th IEEE Conf. on Decision and Control, 1545-1550. Kobe, Japan.

Lovera, M. and Mercère, G. (2007). Identification for gainscheduling: a balanced subspace approach. In Proc. of the American Control Conf., 858-863. New York City, USA.

Scherer, C.W. (1996). Mixed $\mathcal{H}_{2} / \mathcal{H}_{\infty}$ control for timevarying and linear parametrically-varying systems. Int. Journal of Robust and Nonlinear Control, 6(9-10), 929952.

Silverman, L.M. and Meadows, H.E. (1965). Degrees of controllability in time-variable systems. In Proc. of the National Electronics Conf., volume 21, 689-693. Chicago.

Silverman, L.M. and Meadows, H.E. (1967). Controllability and observability in time-variable linear systems. SIAM Journal on Control, 5(1), 64-73.

Tóth, R. (2010). Modeling and Identification of Linear Parameter-Varying Systems. Lecture Notes in Control and Information Sciences, Vol. 403. Springer-Germany.

Tóth, R., Heuberger, P.S.C., and Van den Hof, P.M.J. (2009). Asymptotically optimal orthonormal basis functions for LPV system identification. Automatica, 45(6), $1359-1370$.

Tóth, R., Willems, J.C., Heuberger, P.S.C., and Van den Hof, P.M.J. (2011). The behavioral approach to linear parameter-varying systems. in print, IEEE Trans. on Automatic Control.

Wassink, M.G., van de Wal, M., Scherer, C.W., and Bosgra, O. (2004). LPV control for a wafer stage: Beyond the theoretical solution. Control Engineering Practice, 13(2), 231-245. 